\title{
Influence of Breathing on the Measurement of Lipids in the Myocardium by ${ }^{1} \mathrm{H}$ MR Spectroscopy
}

\author{
M. DROBNY ${ }^{1}$, P. SEDIVY ${ }^{1}$, M. DEZORTOVA ${ }^{1}$, D. WAGNEROVA ${ }^{1}$, M. HAJEK ${ }^{1}$ \\ ${ }^{1}$ MR-Unit, Department of Diagnostic and Interventional Radiology, Institute for Clinical and \\ Experimental Medicine, Prague, Czech Republic
}

Received July 17, 2015

Accepted July 31, 2015

\section{Summary}

The myocardium examination by MR spectroscopy is very challenging due to movements caused by the cardiac rhythm and breathing. The aim of the study was to investigate the influence of breathing on the quantitative measurement of lipid/water ratios in different groups of volunteers and different measuring protocols. We examined the lipid content of myocardium at $3 T$ using the proton single voxel spectroscopy. Three protocols (free breathing, breath hold and the use of respiratory navigator) controlled by ECG were used for the examination of 42 adult volunteers including 14 free divers. Spectra were evaluated using jMRUI software. An average content of lipids in the healthy interventricular septum, gained by all protocols was equal to $0.6 \%$, which is in agreement with other published data. Based on the quality of examinations and the highest technical success, the best protocol seems to be the one containing a respiratory navigator since it is more acceptable by patients. Based on our results and the literature data we can conclude that MR spectroscopy is able to distinguish patients from controls only if their myocardial lipid content is higher than $1.6 \%$ (mean value of lipids plus two standard deviations).

\section{Key words}

Lipid content $\bullet$ Myocardium $\bullet$ Artifacts $\bullet 3 \mathrm{~T} \bullet{ }^{1} \mathrm{H}$ MR spectroscopy

\section{Corresponding author}

M. Dezortova, MR-Unit, Department of Diagnostic and Interventional Radiology, Institute for Clinical and Experimental Medicine, Videnska 1958/9, 14021 Prague 4, Czech Republic. Fax: +420-24172 8224. E-mail: mode@medicon.cz

\section{Introduction}

The first in vivo ${ }^{1} \mathrm{HMR}$ spectra of the myocardium were measured in the early 1990s (Kreis et al. 1998) and so far the majority of data have been obtained at magnetic field 1.5T (Table 1). A typical spectrum of the myocardium shows signals of lipids in the area of aliphatic protons (0.7-2.5 ppm) and water (4.7 ppm) which is used as a standard for the calculation of fat content in the myocardium. Some authors also describe a signal of the methyl group of creatine/ phosphocreatine with the chemical shift of $3.0 \mathrm{ppm}$ and signal at $3.2 \mathrm{ppm}$ corresponding to the protons of the betaine group of water soluble choline-containing compounds (Kreis et al. 1998).

In the heart of adults, $60-80 \%$ of adenosine triphosphate (ATP) comes from beta-oxidation of fatty acids (FA). Esterified FAs are a major source of lipids for human heart. All tissues obtain lipids from free FAs associated with albumin, lipoproteins, and de novo synthesis, which is supposed to play a minor role in heart lipid metabolism. An imbalance between FA uptake and oxidation leads to accumulation of long chain FAs in form of triglycerides (TG) and phospholipids. Redundant lipids, especially TG, are stored in lipid droplets and can cause lipotoxic myopathy. These droplets were found in hearts e.g. of patients suffering from diabetes mellitus and metabolic syndrome (Goldberg et al. 2012).

${ }^{1} \mathrm{H}$ MR spectra of the myocardium may be used to determine the lipid concentration in the cardiac tissue. Fat in myocytes is an early manifestation of the type 2 diabetes mellitus and it is also typical for population suffering from obesity (Rijzewijk et al. 2008, McGavock 
et al. 2006). Previous research has shown that heart failure may be associated with excessive lipolysis and lipotoxic myocytes damage (Sharma et al. 2004). Measurement of fat distribution in the myocardium provides useful information about the degree of its loading and damage.

In most cases, the single voxel spectroscopy (SVS) has been used to acquire ${ }^{1} \mathrm{H}$ MR spectra from the myocardium. The measured volume of interest (VOI) is typically placed on the septum separating the right and left ventricles with the VOI size of several cubic centimeters. The ratio of signal intensity of lipids (Lip, triglycerides) to the unsuppressed water signal is usually used for the quantification. Table 1 summarizes these ratios together with measurement parameters obtained for healthy controls by several authors. Recently, the use of new scanners with a higher magnetic field (3T) and new sequences has had the ability to increase sensitivity and to partly eliminate the influence of some artifacts; nevertheless, the obtained results are very similar to the results obtained at $1.5 \mathrm{~T}$ (Table 1 ). Similar examinations can also be implemented using the MR imaging methods (Liu et al. 2010).

Table 1. Studies concerned the measurement of lipid content in myocardium. According Pubmed up to April 2015.

\begin{tabular}{|c|c|c|c|c|c|c|c|c|c|}
\hline First Author & $\begin{array}{c}\mathbf{B}_{0} \\
{[\mathrm{~T}]}\end{array}$ & Method & Sequence & Technique & $\begin{array}{c}\text { TR } \\
{[\mathrm{ms}]}\end{array}$ & $\begin{array}{c}\text { TE } \\
{[\mathrm{ms}]}\end{array}$ & $\begin{array}{l}\text { VOI } \\
{[\mathrm{ml}]}\end{array}$ & $\begin{array}{c}\text { Relaxation } \\
\text { time } \\
\text { correction }\end{array}$ & $\begin{array}{c}\text { Lipids/water } \\
{[\%]}\end{array}$ \\
\hline Liu 2010 & 3 & MRI & $\begin{array}{c}\text { Dual-echo } \\
\text { SPGR }\end{array}$ & $\mathrm{BH}$ & 6.3 & $\begin{array}{c}2.46 \\
(3.69)\end{array}$ & & $\mathrm{T}_{2}{ }^{*}$ & $1.04 \pm 0.40$ \\
\hline Liu 2010 & 3 & MRS & PRESS & $\mathrm{BH}$ & R-R wave & 30 & & $\mathrm{~T}_{2}^{*}$ & $0.7 \pm 0.5$ \\
\hline Rial 2011 & 3 & MRS & STEAM & $\mathrm{BH}$ & 4000 & 10 & $8-16$ & None & $0.46 \pm 0.20$ \\
\hline Winhofer 2012 & 3 & MRS & PRESS & $\mathrm{BH}$ & $700-1250$ & 30 & 6 & $\mathrm{~T}_{1} \cdot \mathrm{T}_{2}$ & $\begin{array}{c}0.5 \pm 0.4 * \\
(0.20 \pm 0.17)\end{array}$ \\
\hline Krssak 2011 & 3 & MRS & PRESS & $\mathrm{RN}$ & $\begin{array}{l}\text { According } \\
\text { breathing }\end{array}$ & 30 & 6 & $\mathrm{~T}_{1} \cdot \mathrm{T}_{2}$ & $\begin{array}{l}1.0 \pm 0.5 * \\
(0.4 \pm 0.2)\end{array}$ \\
\hline $\begin{array}{l}\text { van der Meer } \\
2007 a\end{array}$ & 1.5 & MRS & PRESS & $\mathrm{RN}$ & $>3000$ & 26 & 8 & None & $0.38 \pm 0.02$ \\
\hline Hammer 2008b & 1.5 & MRS & & $\mathrm{RN}$ & 3000 & 26 & 8 & None & $0.35 \pm 0.14$ \\
\hline $\begin{array}{l}\text { Schrauwen- } \\
\text { Hinderling } 2010\end{array}$ & 1.5 & MRS & PRESS & $\mathrm{RN}$ & 4000 & 26 & 6 & None & $0.99 \pm 0.15$ \\
\hline $\begin{array}{l}\text { van der Meer } \\
2007 \mathrm{~b}\end{array}$ & 1.5 & MRS & PRESS & $\mathrm{RN}$ & $>3000$ & 26 & 8 & None & $0.38 \pm 0.05$ \\
\hline Bilet 2011 & 1.5 & MRS & PRESS & $\mathrm{RN}$ & 4000 & 26 & 6 & None & $0.26 \pm 0.04$ \\
\hline Hammer 2008a & 1.5 & MRS & & $\mathrm{RN}$ & 3000 & 10 & 8 & None & $0.66 \pm 0.09$ \\
\hline Rijzewijk 2008 & 1.5 & MRS & & $\mathrm{RN}$ & $>3000$ & 26 & 8 & None & $0.65 \pm 0.05$ \\
\hline $\begin{array}{l}\text { McGavock } \\
2007\end{array}$ & 1.5 & MRS & PRESS & RN & 4000 & & 6 & None & $0.46 \pm 0.30$ \\
\hline Sai 2013 & 1.5 & MRS & PRESS & FB & $>4000$ & 30 & 2 & None & $0.9 \pm 0.4$ \\
\hline Wu 2012 & 1.5 & MRS & PRESS & $\mathrm{BH}$ & $\begin{array}{c}2 \mathrm{x}(3 \mathrm{x}) \\
\text { R-R wave }\end{array}$ & 35 & 50 & None & $6.7 * *$ \\
\hline Graner 2014 & 1.5 & MRS & PRESS & $\mathrm{RN}$ & $>3000$ & 35 & & None & $0.86(0.31-2.24)$ \\
\hline Petrisch 2012 & 1.5 & MRS & & $\mathrm{RN}$ & 2x R-R wave & 30 & 6 & None & $0.52 \pm 0.11$ \\
\hline
\end{tabular}

$\mathrm{TR}$, repetition time; $\mathrm{TE}$, echo time; VOI, volume of interest; $\mathrm{BH}$, breath hold; $\mathrm{RN}$, respiratory navigator; $\mathrm{FB}$, free breathing; $*$ data without relaxation correction (Krssak et al. 2004), ** data were not used for comparison, the average value gained on 3T (1.5T) scanner is $0.7 \%(0.6 \%)$. 
The aim of our study was to describe the influence of breath holding, controlled breathing and cardiac cycle and some other parameters on the calculation of lipid content in the cardiac tissue and to compare these data with the ones available in the literature. These three different modes of breathing during signal acquisition were tested with volunteers who were able to hold their breath up to several minutes.

\section{Methods}

\section{Subjects}

42 healthy subjects were examined and divided into three groups. In groups A (pilot group of 16 subjects: $14 \mathrm{~m} / 2 \mathrm{f}$, mean age of $30 \pm 11$ years) and B (12 subjects: $9 \mathrm{~m} / 3 \mathrm{f}$, mean age of $29 \pm 12$ years) there were volunteers without any special training and they were able to hold their breathing for $30 \mathrm{~s}$. Group $\mathrm{C}$ consisted of 14 free divers $(13 \mathrm{~m} / 1 \mathrm{f}$, mean age of $37 \pm 8$ years) who were able to hold their breathing up to several minutes. All the examined subjects were informed about the examination protocol, and their written consent was obtained according to the local Ethical Committee rules.

\section{MR imaging and spectroscopy}

The examinations were performed in the supine position at the Siemens Trio $3 \mathrm{~T}$ system equipped with the eight channels surface array coil.

The turbo FLASH (Fast Low Angle Shot) sequence with repetition time/echo time $(\mathrm{TR} / \mathrm{TE})=\mathrm{R}-\mathrm{R}$ wave/1.32 $\mathrm{ms}$ in long and short axes of the heart was used for the positioning of spectroscopic VOI in the interventricular septum (Fig. 1). The localized ${ }^{1} \mathrm{H}$ MR spectroscopy with the PRESS (Point Resolved Spectroscopy) sequence (Work In Progress (WIP) sequence kindly provided by Siemens) was used with the following parameters: 64 acquisitions with water suppression, 4 without water suppression (spectra were summed during measurements), TE $=35 \mathrm{~ms}$ (the shortest possible TE). TR was controlled by ECG (electrocardiography) and navigator sequences and was given as $\mathrm{TR}=\mathrm{n} \cdot \mathrm{bp}+\Delta \mathrm{BH}_{\mathrm{n}}-\Delta \mathrm{BH}_{1}$ where bp is the period of breathing and $\triangle \mathrm{BH}$ is the difference between the start of the acquisition controlled by ECG and the navigator respectively (Fig. 1d). TR equal to double of the heartbeat period was applied in case of protocols without a navigator. Acquisition time depends on breathing and cardiac cycle. In case of free breathing the acquisition time takes approximately two minutes and with respiratory navigator it's over five minutes.

The VOI size was set at $6 \mathrm{ml}$. The homogeneity of the static magnetic field was set by the GREshim shimming method (WIP sequence) and the fine-tuning was done manually. The full width at half maximum (FWHM) of the water signal less than $50 \mathrm{~Hz}$ (magnitude spectra) was considered to be acceptable for the measurement of the Lip/water ratio; spectra with a broader signal of water were excluded from the study.

Three different examination protocols were applied and compared (all protocols included ECG triggering): 1) free breathing (FB); 2) breath-holding $(\mathrm{BH})$; and 3 ) application of respiratory navigator (RN).

\section{Spectra evaluation}

Two evaluation procedures were applied:

a) Spectra were evaluated using jMRUI with the AMARES module. Gaussian-Lorenzian peaks were applied with maxima at $4.7 \mathrm{ppm}$ for water signal and three peaks for lipids signals (at 0.9, 1.2 and $2.0 \mathrm{ppm}$ ). Integral of water was gained from the spectrum with water suppression (Fig. 2a). Signal of lipids was calculated as a summation of individual integrals; however, in most cases only one component with maximum at $1.2 \mathrm{ppm}$ was detected due to the quality of spectra (there was no detailed structure to recognize) (Fig. 2a).

b) Another subroutine of jMRUI was used for the calculation of magnitude spectra (Fig. 2b). Signal intensities of water and lipids were calculated from the FWHM and height (de Graaf 2007) assuming the Lorentzian shape of the peaks. Application of magnitude spectra solves the problem of signal phasing, which is not easy in some cases of real spectra, especially in the case of spectra where water suppression pulses are used, due to the different phase of the residual signal of water (Fig. 3).

\section{Results}

A summary of results of all measurements is shown as ratios of lipid signals to the water signal (Table 2). No corrections to $\mathrm{T}_{1}$ and $\mathrm{T}_{2}$ were applied. Data in Table 2 also compare results obtained from real and magnitude spectra. The quality of magnitude spectra was sufficient only for group $\mathrm{C}$; in the other cases, the signal of lipids was too broad for the processing (Fig. 2b). 


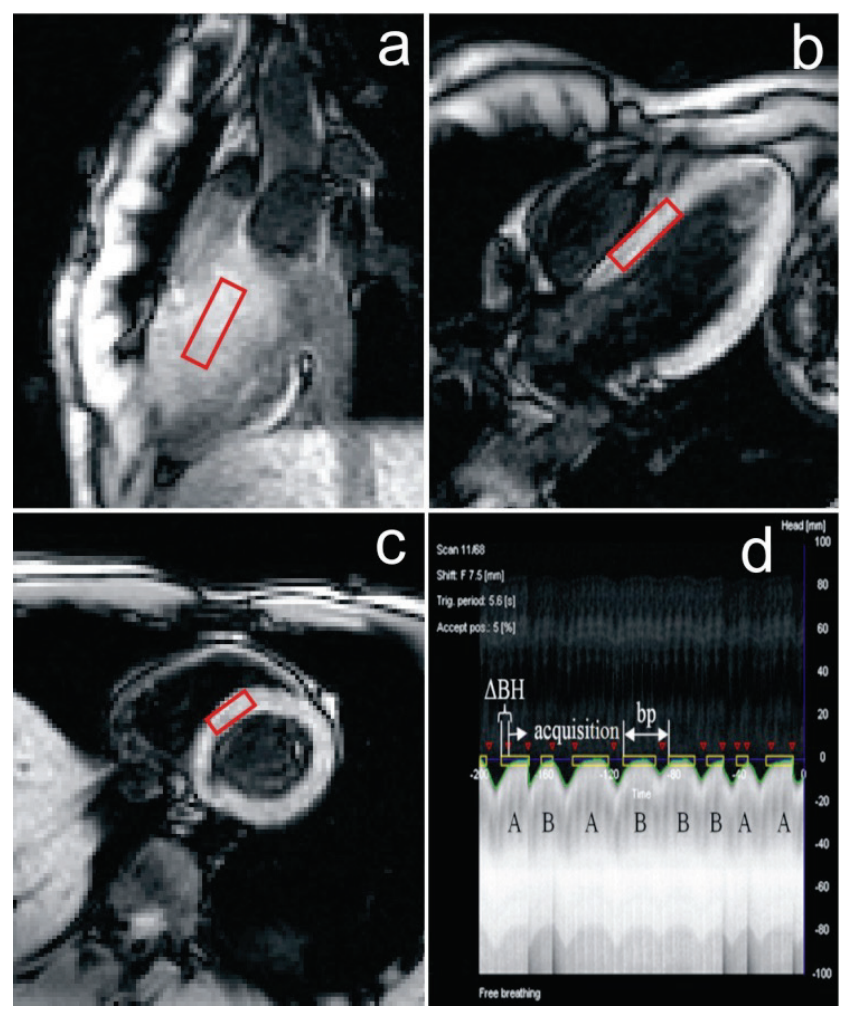

Fig. 1. Orientation of VOI (size $6 \mathrm{ml}$ ) in cardiac axes - long axes (a) and (b) and short axis (c) - measured by turbo FLASH sequence. Screenshot with a record of diaphragm's position in time by respiratory navigator is shown in part $\mathbf{d}$ of the picture. Sequence waits for the moment when the position of diaphragm (green line) reaches the yellow rectangle and the sequence starts when the first ECG signal (red triangular) comes. Letters A in the figure mean that condition of double-triggered sequence is fulfilled and acquisition starts. In case B condition is not fulfilled.

The technical success of the measurement is primarily given by the experience of the operator and motion artifacts which can be eliminated or even dismissed by using ECG. Our technical success of the examination was $56 \%$ in group A, $100 \%$ in case of group B and $79 \%$ and $71 \%$ in group C. We can see that also respiratory navigator triggering can significantly improve the quality of the spectra.

One subject was examined repeatedly by the protocol with the respiratory navigator. The reproducibility measured from the subject was found to be approximately $13 \%$ (ratio of standard deviation and mean value).
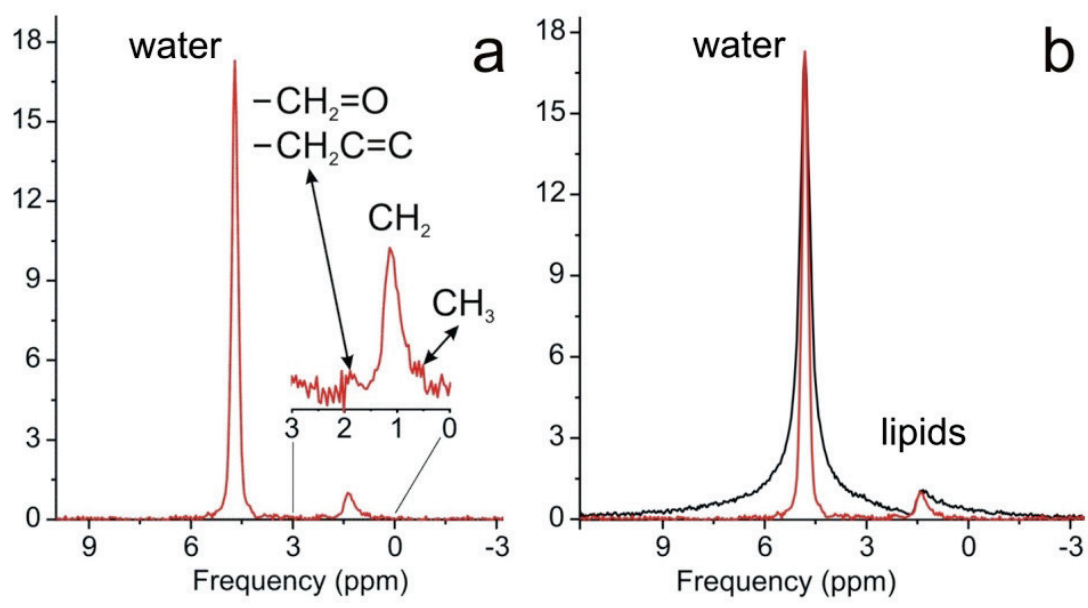

Fig. 2. In vivo spectrum (lipids area at $0-3 \mathrm{ppm}$ is highlighted) with water suppression (a) and comparison of real (red line) and magnitude (black line) MR spectrum (b).
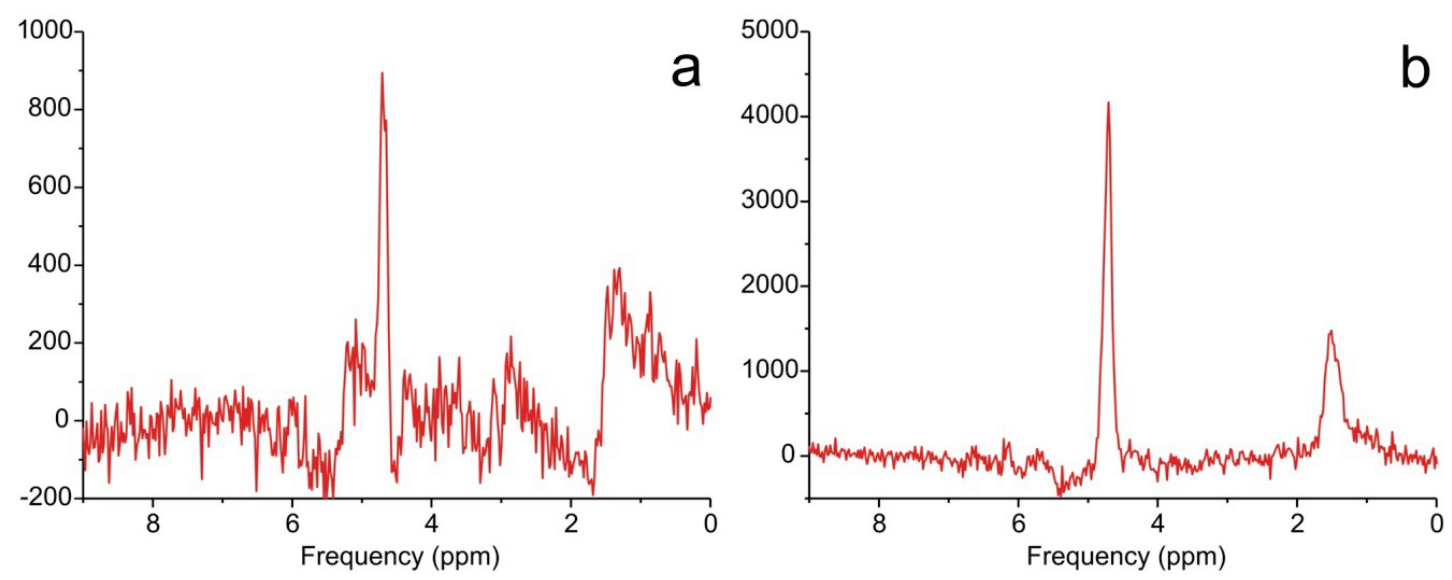

Fig. 3. Comparison of spectra with water suppression measured by free breathing (a) and by respiratory navigator (b) protocol (same person). 
Table 2. Lipid content (mean \pm SD) obtained by different ${ }^{1} \mathrm{H}$ MR spectroscopy examination protocols.

\begin{tabular}{lcccccc}
\hline & Group A & \multicolumn{2}{c}{ Group B } & \multicolumn{2}{c}{ Group C } & Total \\
\hline Examination protocol & $\mathrm{FB}$ & $\mathrm{FB}$ & $\mathrm{RN}$ & $\mathrm{BH}$ & $\mathrm{RN}$ & \\
$N / n$ & $16 / 9$ & $12 / 12$ & $12 / 12$ & $14 / 11$ & $14 / 10$ & $68 / 54$ \\
Technical success $\%$ & 56 & 100 & 100 & 79 & 71 & 79 \\
Mean $\pm S D$ & $0.6 \pm 0.4$ & $0.5 \pm 0.3$ & $0.6 \pm 0.4$ & $0.6 \pm 0.5$ & $0.7 \pm 0.3$ & \\
\hline & \multicolumn{7}{c}{ Absolute spectra } & & & \\
\hline N/n & & & $14 / 11$ & $14 / 9$ & $28 / 20$ \\
Technical success $\%$ & & & 79 & 64 & 71 \\
Mean $\pm S D$ & & & $1.0 \pm 0.7$ & $1.1 \pm 0.7$ & \\
\hline
\end{tabular}

$\mathrm{FB}$, free breathing with the ECG signal triggering; $\mathrm{BH}$, breath-holding with the ECG; RN, application the respiratory navigator and the ECG; $N$, total examined subjects; $n$, successfully examined subjects. There are no significant differences among groups and protocol according to ANOVA ( $p=0.73$ or 0.85 with group $A$, resp.).

\section{Discussion}

To the best of our knowledge, we have found only 15 papers focused on the measurement of fat content in the myocardial tissue with healthy control values. Published data (without relaxation times correction) for the $1.5 \mathrm{~T}$ examination show the mean value of the Lip/water ratio to be $0.6 \%$; the results of the $3 \mathrm{~T}$ examinations indicate $0.7 \%$. These values are not significantly different because of high data variation. In our experience, two main factors influence the final value of the ratio. The first one is motion artifact due to heartbeat and breathing which significantly changes local shim, the second factor is the saturation effect due to $T_{1}$ and $T_{2}$ relaxation times.

The technical design of our protocol is similar to protocols described in other papers (Rial et al. 2011, Winhofer et al. 2012, Schrauwen-Hinderling et al. 2010); nevertheless, the authors do not describe the influence of breathing in detail.

Our comparison of protocols shows that spectra with the double triggering sequence (ECG and respiratory navigator) provide the best quality and can be used as a routine protocol of myocardium examination because the position of the myocardium is more stable and exactly defined during the examination (Fig. 3). It corresponds to the fact that water suppression procedure is more effective with regard to this protocol due to better phasing. A very important part of such measurement is the shimming procedure. Using the GREshim sequence brought improvement to the quality of the obtained spectra according to reached FWHM.
It is in agreement with other authors who used double triggered sequences (Krssak et al. 2011, Hammer et al. 2008a, McGavock et al. 2007) and obtained similar results and accuracy as in our case (Table 1). However, there is still demand for further improvement in minimizing the movement artifacts and improvement of the magnetic field homogeneity in the investigated area which is influenced by blood stream in the vicinity.

Reduction of the $T_{1}$ saturation effect is possible by using at least a doubled cardiac period for triggering which increases TR. Another effect that can influence the result of the measurement is echo time which should be used as short as possible ( $\mathrm{TE}=10$ to $35 \mathrm{~ms}$ in the published studies). Correction for relaxation times $T_{2}$ and $T_{1}$ is not considered in most of these studies because the direct measurement of the relaxation times is difficult to perform. Up to now, $T_{2}$ and $T_{1}$ corrections in myocardium spectra were only done with relaxation times obtained in skeletal muscles (Krssak et al. 2004) in studies by Winhofer et al. (2012) and Krssak et al. (2011). This correction reduces the Lip/water ratio by a factor of approximately 2.5 .

We have not used corrections for $T_{1}$ and $T_{2}$ and our results summarized in Table 2 are in the range of the published data for healthy subjects examined at 3T (Liu et al. 2010, Rial et al. 2011, Winhofer et al. 2012, Krssak et al. 2011). ANOVA statistical tests (Analysis Of Variance) with Bonferroni correction show no differences in the lipid content between groups of volunteers A, B and C and examination protocols.

It is known that some pathologies (mostly connected with diabetes mellitus) are associated with 
a higher cardiac lipid content (McGavock et al. 2007). As the results are affected by a rather high standard deviation, the practical use of the method in clinical practice must take this fact into consideration. Our data show that the content of fat to be considered out of controls must be higher than $1.6 \%$, i.e. mean value of the lipid content in healthy controls (i.e. $0.6 \%$ ) plus a doubled standard deviation (probability of $95 \%$ ). On a pilot group of six patients diagnosed with cardiomyopathy and diabetes mellitus type 2 were gained values over our set limit.

Nowadays, even with very high level technical facilities, measurement of myocardial lipid content at $3 \mathrm{~T}$ still remains challenging. The use of respiratory navigator improves the spectra quality, but it does not affect the final lipid value determination. Nevertheless, based on our results and the literature it can be concluded that useful clinical data can be obtained for subjects with a lipid content higher than $1.6 \%$, which represents the mean value of lipid content plus two standard deviations.

\section{Conflict of Interest}

There is no conflict of interest.

\section{Acknowledgements}

The study was supported by projects IGA MH CR NT11275-6, MHCZ-DRO 00023001 IKEM. Approval of this study was given by local ethical committee. Clinical protocols are certified according to the ISO 9001:2008 norm by the local ethical committee. The guarantor of the study is Dr. Milan Hajek.

\section{Abbreviations}

FA, fatty acid; SVS, single voxel spectroscopy; VOI, volume of interest; Lip, lipids; FLASH, fast low angle shot; TE, echo time; TR, repetition time; PRESS, point resolved spectroscopy; WIP, work in progress; FWHM, full width at half maximum; ECG, electrocardiography; $\mathrm{BH}$, breath hold; $\mathrm{FB}$, free breathing; $\mathrm{RN}$, respiratory navigator; ANOVA, analysis of variance.

\section{References}

BILET L, VAN DE WEIJER T, HESSELINK MK, GLATZ JF, LAMB HJ, WILDBERGER J, KOOI ME, SCHRAUWEN-HINDERLING VB: Exercise-induced modulation of cardiac lipid content in healthy lean young men. Basic Res Cardiol 106: 307-315, 2011.

DE GRAAF RA: In Vivo NMR Spectroscopy: Principles and Techniques. 2nd edition. John Wiley \& Sons, Chichester, 2007.

GOLDBERG IJ, TRENT CM, SCHULZE PC: Lipid metabolism and toxicity in the heart. Cell Metab 15: 805-812, 2012.

GRANER M, SIREN R, NYMAN K, LUNDBORN J, HAKKARAINEN A, PENTIKAINEN MO, LAUERMA K, LUNDBORN N, ADIELS M, NIEMINEN MS, TASKINEN MR: Cardiac steatosis associates with visceral obesity in nondiabetic obese men. J Clin Endocrinol Metab 98: 1189-1197, 2013.

GRANER M, PENTIKAINEN MO, NYMAN K, SIREN R, LUNDBORN J, HAKKARAINEN A, LAUERMA K, LUNDBORN N, NIEMINEN MS, PETZOLD M, TASKINEN MR: Cardiac steatosis in patients with dilated cardiomyopathy. Heart 100: 1107-1112, 2014.

HAMMER S, VAN DER MEER RW, LAMB HJ, DE BOER HH, BAX JJ, DE ROOS A, ROMIJN JA, SMIT JW: Shortterm flexibility of myocardial triglycerides and diastolic function in patients with type 2 diabetes mellitus. $A m$ J Physiol Endocrinol Metab 295: 714-718, 2008a.

HAMMER S, VAN DER MEER RW, LAMB HJ, SHAER M, DE ROOS A, SMIT JW, ROMIJN JA: Progressive caloric restriction induces dose-dependent changes in myocardial triglyceride content and diastolic function in healthy men. J Clin Endocrinol Metab 93: 497-503, 2008 b.

KREIS R, FELBLINGER J, JUNG B, BOESCH C: In vivo 1H-MR spectroscopy of the human heart. MAGMA 6: 164-167, 1998.

KRSSAK M, MLYNARIK V, MEYERSPEER M, MOSER E, RODEN M: 1H NMR relaxation times of skeletal muscle metabolites at 3 T. MAGMA 16: 155-159, 2004.

KRSSAK M, WINHOFER Y, GOEBL C, BISCHOF M, REITER G, KAUTZKY-WILLER A, LUGER A, KREBS M, ANDERWALD C: Insulin resistance is not associated with myocardial steatosis in women. Diabetologia 54: 1871-1878, 2011. 
LIU CY, REDHEUIL A, OUWERKERK R, LIMA JAC, BLUEMKE DA: Myocardial fat quantification in humans: evaluation by two-point water-fat imaging and localized proton spectroscopy. Magn Reson Med 63: 892-901, 2010.

MCGAVOCK JM, VICTOR RG, UNGER RH, SZCZEPANIAK LS: Adiposity of the heart, revisited. Ann Intern Med 144: 517-524, 2006.

MCGAVOCK JM, LINGVAY I, ZIB I, TILLERY T, SALAS N, UNGER R, LEVINE BD, RASKIN P, VICTOR RG, SZCZEPANIAK LS: Cardiac steatosis in diabetes mellitus: a 1H-magnetic resonance spectroscopy study. Circulation 116: 1170-1175, 2007.

PETRITSCH B, KOESTLER H, MACHANN W, HORN M, WENG AM, GOLTZ JP, HAHN D, NIEMANN M, WEIDEMANN F, WANNER C, BEER M: Non-invasive determination of myocardial lipid content in Fabry disease by 1H-MR spectroscopy. Rofo 184: 1020-1025, 2012.

RIAL B, ROBSON MD, NEUBAUER S, SCHNEIDER JE: Rapid quantification of myocardial lipid content in humans using single breath-hold 1H MRS at 3 Tesla. Magn Reson Med 66: 619-624, 2011.

RIJZEWIJK LJ, VAN DER MEER RW, SMIT JW, DIAMANT M, BAX JJ, HAMMER S, ROMIJN JA, DE ROOS A, LAMB HJ: Myocardial steatosis is an independent predictor of diastolic dysfunction in type 2 diabetes mellitus. Am J Physiol Endocrinol Metab 52: 1793-1799, 2008.

SAI E, SHIMADA K, YOKOYAMA T, SATO S, MIYAZAKI T, HIKI M, TAMURA Y, AOKI S, WATADA H, KAWAMORI R, DAIDA H: Association between myocardial triglyceride content and cardiac function in healthy subjects and endurance athletes. PLoS One 8: e61604, 2013.

SCHRAUWEN-HINDERLING VB, HESSELINK MK, MEEX R, VAN DER MADE S, SCHAER M, LAMB H, WILDBERGER JE, GLATZ J, SNOEP G, KOOI ME, SCHRAUWEN P: Improved ejection fraction after exercise training in obesity is accompanied by reduced cardiac lipid content. J Clin Endocrinol Metab 95 : 1932-1938, 2010.

SCHRAUWEN-HINDERLING VB, MEEX RCR, HESSELINK MKC, VAN DE WEIJER T, LEINER T, SCHAER M, LAMB HJ, WILDBERGER JE, GLATZ JFC, SCHRAUWEN P, KOOI ME: Cardiac lipid content is unresponsive to a physical activity training intervention in type 2 diabetic patients, despite improved ejection fraction. Cardiovasc Diabetol 10: 47, 2011.

SHARMA S, ADROQUE JV, GOLFMAN L, URAY I, LEMM J, YOUKER K, NOON GP, FRAZIER OH, TAEGTMEYER H: Intramyocardial lipid accumulation in the failing human heart resembles the lipotoxic rat heart. FASEB J 18: 1692-1700, 2004.

VAN DER MEER RW, DOORNBOS J, KOZERKE S, SCHAER M, BAX JJ, HAMMER S, SMIT JW, ROMIJN JA, DIAMANT M, RIJZEWIJK LJ, DE ROOS A, LAMB HJ: Metabolic imaging of myocardial triglyceride content: reproducibility of $1 \mathrm{H}$ MR spectroscopy with respiratory navigator gating in volunteers. Radiology 245: 251-257, 2007a.

VAN DER MEER RW, HAMMER S, SMIT JW, FROELICH M, BAX JJ, DIAMANT M, RIJZEWIJK LJ, DE ROOS A, ROMIJN JA, LAMB HJ: Short-term caloric restriction induces accumulation of myocardial triglycerides and decreases left ventricular diastolic function in healthy subjects. Diabetes 56: 2849-2853, $2007 \mathrm{~b}$.

WINHOFER Y, KRSSAK M, JANKOVIC D, ANDERWALD CH, REITER G, HOFER A, TRATTNIG S, LUGER A, KREBS M: Short-term hyperinsulinemia and hyperglycemia increase myocardial lipid content in normal subjects. Diabetes 61: 1210-1216, 2012.

WU J, YANG L, YANG J, YANG CH, ZHANG XH: Gender-specific normal levels of myocardial metabolites determined by localized 1H-magnetic resonance spectroscopy. J Int Med Res 40: 1507-1512, 2012. 